\title{
Linear morphological stability analysis of the solid-liquid interface in rapid solidification of a binary system
}

\author{
P. K. Galenko* ${ }^{1}$ and D. A. Danilov** \\ *German Aerospace Center, Institute of Space Simulation, 51170 Cologne, \\ Germany \\ **University of Applied Sciences, 76133 Karlsruhe, Germany
}

\begin{abstract}
The interface stability against small perturbations of the planar solid-liquid interface is considered analytically in linear approximation. Following the analytical procedure of Trivedi and Kurz (Trivedi R, Kurz W. Acta Metall 1986;34:1663), which is advancing the original treatment of morphological stability by Mullins and Sekerka (Mullins WW, Sekerka RF. J Appl Phys 1964;35:444) to the case of rapid solidification, we extend the model by introducing the local nonequilibrium in the solute diffusion field around the interface. A solution to the heat- and masstransport problem around the perturbed interface is given in the presence of the local nonequilibrium solute diffusion. Using the developing local nonequilibrium model of solidification, the self-consistent analysis of linear morphological stability is presented with the attribution to the marginal (neutral) and absolute morphological stability of a rapidly moving interface. Special consideration of the interface stability for the cases of solidification in negative and positive thermal gradients is given. A quantitative comparison of the model predictions for the absolute morphological stability is presented with regard to experimental results of Hoglund and Aziz (Hoglund DE, Aziz MJ. Mat Res Soc Symp Proc 1992;205:325) on critical solute concentration for the interface breakdown during rapid solidification of $\mathrm{Si}-\mathrm{Sn}$ alloys. PACS: 05.70.Fh; 47.20.Ma; 81.10.Aj; 81.30.Fb
\end{abstract}

Key words: Interface stability; Solute diffusion; Local nonequilibrium; Undercooling; Directional solidification

$\overline{1 \text { Fax: }}++49-2203-601-2255$; e-mail: Peter.Galenko@dlr.de 


\section{Introduction}

In the solidification of liquids, an initial solid-liquid interface is subject to unstable growth which leads to various crystal patterns of cellular, dendritic, banded, fractal, etc. morphologies $[1,2,3,4,5]$. A scheme of changing the crystal morphology with the interface velocity can be considered in the example of one-phase solidification, i.e. when the liquid transfers into solid without precipitation of additional phases. Figure 1 shows schematically the steady-state growth morphologies which form in a liquid as a result of morphological instability at given interface velocity, $V$, in single-phase solidification. With a small velocity, an initially smooth interface remains planar up to a velocity equal to the critical velocity, $V_{C}$, defined by the constitutional undercooling [6]. Behind $V_{C}$, the smooth interface becomes unstable and the interface exhibits a steady cellular morphology. By further increasing the velocity, a surface of cells may become unstable with the developing of dendritic patterns. At high interface velocity, dendritic patterns degenerate with the appearing of rapidly moving cells. A demarcation line at $V=V_{A}$ divides the regions between the interface instability, $V<V_{A}$, and the absolute stability, $V>V_{A}$, where the planar interface is morphologically stable against small perturbations of its form. This demarcation line is usually known as the critical velocity, $V_{A}$, for absolute stability of the planar interface. The sequence of growth morphologies, Fig. 1, is well-known from experiments on directional solidification and solidification in the undercooled state [7]. It has been demonstrated in computational modeling [8] of crystal growth as well.

The theory of morphological stability was developed first by Mullins and Sekerka, who considered the stability of a spherical particle grown into a supersaturated solution [9], and stability of the planar front during directional solidification of a binary liquid [10]. In investigating the evolution of small harmonic perturbations of the planar interface [10], they provided a rigorous basis of linear morphological (in)stability at low solidification velocity. Particularly, Mullins and Sekerka introduced a concept of marginal stability for the wavelength of perturbation, which gives neutral stability of the plane when the amplitude of perturbation does not change in time. Behind the critical velocity of the marginal stability, the increasing of the amplitude of perturbation in time may lead to cellular or dendritic interfaces. The theory [10] gave rise to a great number of investigations of morphological transformations due to linear instability of interfaces and nonlinear behaviour of unstable interfaces. These are presented in overview by Coriell and McFadden [11] and monograph by Davis [12].

In its classic form [10], the theory assumes a local equilibrium at the interface, which is an excellent approximation for many metallic systems solidifying at small interface velocities. At large driving force for the interface advancing, 
and with the increasing of its velocity, the analysis of Mullins and Sekerka can be modified. Trivedi and Kurz [13] extended the analysis of Mullins and Sekerka [10] for the case of rapid solidification, and introduced the stability functions dependent on the interface velocity. By taking into account the velocity dependent coefficient of solute redistribution (partitioning function), they developed an analytical model [14] of microstructure formation under directional solidification, over the range from low interface velocity up to velocity, $V_{A}$, of absolute morphological stability.

In addition to the previous analysis of pattern formation and morphological stability of the interface in which the treatment is extended to rapid solidification [13] and nonequilibrium effects at the interface [14], the local nonequilibrium in bulk phases may play an essential role in rapid solidification. Particularly, a deviation from local equilibrium in solute diffusion may act on the rapid advancing of the solid-liquid interface because the interface velocity, $V$, can be of the order or even greater than the solute diffusion speed, $V_{D}$, in bulk phases. For instance, the diffusion speed can be of the order of [15]: $V_{D} \sim 0.1-10 \mathrm{~m} / \mathrm{s}$. In modern experiments on solidification of undercooled droplets the interface velocity approaches [16]: $V=10-100 \mathrm{~m} / \mathrm{s}$. Therefore, the undercooling of liquids is sufficient for detecting solidification with the interface velocity comparable to the diffusion speed.

Considering the process of solute diffusion, Fick's first law is obtained on the basis of classic irreversible thermodynamics of Onsager and Prigogine which assumes propagation of concentration disturbances with an infinite speed and with local equilibrium in the bulk phases [17]. Local equilibrium is characterized by the statistical distribution function, given by the first order term of its expansion [18]. However, for a high-velocity solidification front, the time for crystallizing of a local volume is comparable to the time for relaxation of the diffusion flux to its steady-state value [15]. In this case, local equilibrium is absent in the bulk phases and the solute flux cannot be described by the classical Fick's first law. Including the evolution equation for the diffusion flux, the analysis of Galenko and Sobolev [19] shows that the deviations from local equilibrium in phases and at the interface drastically affect both the solute diffusion and the interface kinetics. Therefore, in this article we consider the linear stability analysis for a rapidly moving interface under local nonequilibrium solute diffusion.

The linear morphological analysis of the interface stability has been developed and used to obtain a stable tip of dendrite growing under local nonequilibrium solute diffusion in rapid solidification [20]. A marginal stability criterion was used and concluded that at $V=V_{D}$ the complete transition to diffusionless solidification may proceed sharply, with the appearing of the break point in the kinetic curves "dendrite velocity - undercooling" and "dendrite tip radius - undercooling". In addition to this, Lee et al. [21] performed the 
linear stability analysis in rapid directional solidification using the model of Galenko and Sobolev [19]. As they showed [21], the effect of the local nonequilibrium in solute diffusion postpones the onset of the cellular instability in better agreement with experimental data, in comparison with the model predictions in which the local nonequilibrium only at the interface is considered. However, the analysis of Lee et al. [21] was not self-consistent (in the analysis, the authors used an expression for the slope of kinetic liquidus obtained from the local equilibrium thermodynamics). As it has been noted in Ref. [20], the predictions of rapid solidification of alloys can be satisfactory compared with experimental data only on the basis of self-consistent model (i.e., when all model functions are taking into account the deviation from local equilibrium in the solute diffusion field). Consequently, the first purpose of the present article is to analyze the morphological stability of the planar interface on the basis of the self-consistent model of local nonequilibrium solidification.

In the present analysis, special consideration is paid to the stability of the interface around the demarcation line, $V=V_{A}$, below which the interface instability occurs and behind of which the absolute morphological stability proceeds, Fig. 1. This transition is known from experiment as a transition from cellular patterns to the segregation-free patterns $[22,23]$. The crystal microstructure after the transition is shown in Fig. 2. As it has been stated [24], the transition from the macroscopically smooth solid-liquid interface to the cellular-dendritic microstructures occurs with the decreasing of the interface velocity, $V$, below critical velocity, $V_{A}$, for absolute stability of the planar front. Around the velocity $V=V_{A}$, the kinetics of crystal growth begin to disagree with the predictions of the model in which the local nonequilibrium only at the interface is considered (see the results of analysis in Ref. [20]). Consequently, the second purpose of this article is a quantitative evaluation of the discrepancy between the present local nonequilibrium model and the model in which the local nonequilibrium only at the interface is taken. It is also in comparison with experimental data on morphological stability of the interface in solidifying alloy.

The article is organized as follows. In Sec. 2 we give a formal description of departure from local equilibrium due to solute diffusion and give a set of governing equations to analyze the morphological stability of the interface. In Sec. 3 we analyze the influence of perturbations in fields and at the interface on the linear stability of the planar interface. In Sec. 4, an obtained criterion of marginal (neutral) stability allows us to analyze the morphological stability of the interface for the case of solidification an undercooled melt (with negative temperature gradient) and for the directional solidification (with the positive temperature gradient). The absolute stability of the planar interface is analyzed in Sec. 5. A discussion about expressions for solute trapping and kinetic liquidus which define a final form of the function for the absolute stability of the planar interface is given in Sec. 6. Also, in this section, we compare 
the derived function for the absolute interface stability with the available experimental results obtained in Si-Sn alloy solidification. Finally, in Sec. 7 we present a summary of our conclusions.

\section{Statement of the problem}

We shall consider a dilute binary alloy that undergoes nonisothermal solidification in infinite space. Let us take into account the heat diffusion in phases, solute diffusion in the liquid, and one can neglect the solute diffusion in solid. The main physical assumption of the present problem is an absence of local equilibrium both at the solid-liquid interface and in the solute diffusion field around the interface. In this case, the degree of local nonequilibrium is estimated by the relation of the interface velocity, $V$, and the diffusion speed, $V_{D}$, which is a parameter of the process of diffusion and can have different values at the interface and in bulk phases. The speed $V_{D}$ is a maximum speed of propagation of the diffusion profile in the system and defined as $V_{D}=\left(D / \tau_{D}\right)^{1 / 2}$, where $D$ is the diffusion coefficient, and $\tau_{D}$ is the time of relaxation of diffusion flux to its steady-state value. Therefore, we develop the rapid solidification model which is taking into account the finiteness of the diffusion speed in the system.

\subsection{Departure from local equilibrium}

If local thermodynamic equilibrium in the bulk is not reached, the connection between the vectors of diffusion fluxes, $\mathbf{q}_{i}$ and $\mathbf{J}$, and the driving forces, $\nabla T_{i}$ and $\nabla C$, for the heat and solute diffusion, respectively, have the following integral form:

- relaxation of the heat flux

$$
\mathbf{q}_{i}(\mathbf{r}, t)=-\int_{-\infty}^{t} D_{q}^{i}\left(t-t^{*}\right) \nabla T_{i}\left(t^{*}, \mathbf{r}\right) d t^{*}
$$

- relaxation of the solute diffusion flux

$$
\mathbf{J}(\mathbf{r}, t)=-\int_{-\infty}^{t} D_{j}\left(t-t^{*}\right) \nabla C\left(t^{*}, \mathbf{r}\right) d t^{*}
$$

where index $i=L$ or $i=S$ is related to the liquid or solid phases, respectively. $T_{i}$ are the temperatures in the phases, $C$ is the solute concentration in the liquid, $t$ is the time, $\mathbf{r}$ is the radius-vector of a point in the system, and $D_{R}\left(t-t^{*}\right)$ are the relaxation functions of the fluxes $(R=q$ or $R=j)$. Equations (1) and (2) imply the fact that when the interface moves with a 
high velocity, local equilibrium in the fields does not occur and the diffusion fluxes at a point in the system no longer depend on the instantaneous gradients of the temperature and chemical composition, but are also determined by the local prehistory of the solidification process.

Equations (1) and (2) represent general expressions for evolution prehistory of the diffusion processes. For the case of heat diffusion, when the heat propagates with much higher speed in comparison with the interface velocity, the influence of local nonequilibrium in the temperature field on the kinetics of the interface advancing is negligible (see the analysis of heat transfer in rapid solidification in Ref. [25]). Therefore we specially define the relaxation functions $D_{R}$ in Eqs. (1) and (2) for the important class of dissipative hyperbolic models in which they take the following forms

$$
\begin{gathered}
D_{q}^{i}\left(t-t^{*}\right)=D_{q}^{i}(0) \delta\left(t-t^{*}\right), \\
D_{j}\left(t-t^{*}\right)=D_{j}(0) \exp \left(-\frac{t-t^{*}}{\tau_{D}}\right),
\end{gathered}
$$

where $D_{q}^{i}(0)=K_{i}$ are the thermal conductivity in the liquid $(i=L)$ and solid $(i=S), \delta$ is the Dirac delta-function, and $D_{j}(0)=D / \tau_{D}$ is a value of relaxation function for solute diffusion at a moment $t=t^{*}$.

Eq. (3) describes an instant relaxation which occurs at a moment $t=t^{*}$. Therefore, one can expect a description of local equilibrium heat transport by the function (3) in combination with Eq. (1). In contrast to this, the relaxation function (4) with the flux prehistory (2) leads to local nonequilibrium solute diffusion. In usual circumstances, the relaxation time, $\tau_{D}$, for diffusion flux is very small and the relaxation effects are negligible. It is usual to speak in this case of "viscous diffusion" which can be accurately described by the Fickian local equilibrium approximation. However, equations (2) and (4) describe relevant difference of solute diffusion with respect to the classical Fick's law. To date, departures from this law are known as the appearance of internal effects, couplings of diffusion and viscosity, and longitudinal diffusion (see Refs. $[26,27,28,29]$ and references therein). In addition to these appearances, Eqs. (2) and (4) can also be applied to the process of the phase transition in a strongly nonequilibrium medium. Rapid solidification of a binary melt is a good example of such a nonequilibrium phase transition in which the interface can move with the high velocity comparable with the diffusive speed, $V \sim V_{D} \sim 0.1-10(\mathrm{~m} / \mathrm{s})[15]$. In this case, the time for crystallizing of local bulk is of the order of the relaxation time of the solute diffusion flux [15,19,20], characterizing its decay forward of its local equilibrium value. Therefore when $V \sim V_{D}$, the relaxation interacts with the diffusion process directly, and it is necessary to take into account the local prehistory of the solute diffusion, e.g. in a form described by Eqs. (2) and (4). 
Eq. (4) simulates a physically reasonable situation in which exponential decay of the diffusion flux occurs in the local bulk of the liquid phase. This equation provides the lowest order of approximation of the diffusion flux relaxation. Indeed, substituting Eqs. (3) and (4) for Eqs. (1) and (2), respectively, one obtains

$$
\begin{gathered}
\mathbf{q}_{i}+K_{i} \nabla T_{i}=0, \\
\tau_{D} \frac{\partial \mathbf{J}}{\partial t}+\mathbf{J}+D \nabla C=0 .
\end{gathered}
$$

Equation (5) is a well-known Fourier law which is true for infinite thermal speed in the system, i.e. the heat diffusion flux is instantly relaxed to its local equilibrium value and the effects of local nonequilibrium in the thermal field are negligible. Equation (6) can be treated as the simplest generalization of the classical Fick's first law $\mathbf{J}+D \nabla C=0$ that is recovered when $\tau_{D}=0$ or in stationary situations in which $\partial \mathbf{J} / \partial t=0$. The evolution equation (6) takes into account the relaxation to local equilibrium of the mass flux and is known as the Maxwell-Cattaneo equation in the context of heat transport $[27,28,29]$. By taking the relaxation (2) with the exponential law (4), it follows from Eq. (6) that the flux $\mathbf{J}$ at a point in the system is defined by the evolution of the concentration gradient $\nabla C\left(t^{*}, \mathbf{r}\right)$ during the period $t-\tau_{D}<t^{*}<t$, but not by the gradient $\nabla C(t, \mathbf{r})$ at the moment $t$, as in local equilibrium approximation. Thus, taking into account the exponential decay for the relaxation of the diffusion flux (see Eq. (4)), the simplest evolution equation (6) is obtained for the interaction of the relaxation process and solute diffusion. Instead of Eq. (6), by taking suitable relations for the functions of $D(C), D(\partial C / \partial t)$, or $D(\nabla C)$, one can describe more complicated situations for non-Fickian diffusion in nonequilibrium media. These are described by the evolution equation with the higher order time derivatives or couplings of relaxation to non-local effects for transient processes [28].

The heat and solute diffusion are governed by the balance laws

$$
\begin{gathered}
\chi_{i} \frac{\partial T_{i}}{\partial t}+\nabla \cdot \mathbf{q}_{i}=0, \\
\frac{\partial C}{\partial t}+\nabla \cdot \mathbf{J}=0,
\end{gathered}
$$

where $\chi_{i}$ are the heat capacities in phases. Substitution of Eqs. (5) and (6) into Eqs. (7) and (8), respectively, gives the following system of equations

$$
\begin{gathered}
\frac{\partial T_{i}}{\partial t}=a_{i} \nabla^{2} T_{i} \\
\tau_{D} \frac{\partial^{2} C}{\partial t^{2}}+\frac{\partial C}{\partial t}=D \nabla^{2} C
\end{gathered}
$$


where $a_{i}$ are the thermal diffusivities in the liquid $(i=L)$ and solid $(i=S)$. Equation (9) is the common partial differential equation of parabolic type for the heat transfer which adopts the infinite thermal speed. Equation (10) shows that Eqs. (6) and (8) give rise to the partial differential equation of a hyperbolic type for the solute concentration, which is the simplest mathematical model combining the diffusive (dissipative) mode and the propagative (wave) mode of mass transport under local nonequilibrium conditions. In such a case, Eq. (10) describes the transport process under non-Fickian diffusion.

After integration of Eqs. (9) and (10) over an infinitesimal zone that includes the interface, the following boundary conditions for the diffusion transport hold

$$
\begin{gathered}
-K_{L} \nabla_{n} T_{L}+K_{S} \nabla_{n} T_{S}=Q V_{n}, \\
-D \nabla_{n} C=\left(C-C_{S}\right) V_{n}+\tau_{D} \frac{\partial}{\partial t}\left(\left(C-C_{S}\right) V_{n}\right),
\end{gathered}
$$

where $Q$ is the latent heat of solidification, $\nabla_{n} T_{i}$ and $\nabla_{n} C$ are the normal gradients of temperature and solute concentration to the interface, respectively, $V_{n}$ is the normal velocity of the interface, $C_{S}$ is the solute concentration at the interface in the solid phase given by expression

$$
C_{S}=k C,
$$

and $k$ is the coefficient of solute partitioning at the interface.

\subsection{Governing equations}

Under the assumptions drawn in Section 2.1 we consider the governing equations for analysis of the morphological stability of the planar interface against small perturbations of its form. Our analysis is based on the analysis given by Trivedi and Kurz [13] which is advancing the treatment of Mullins and Sekerka [10] to the case of rapid solidification.

The analysis of interface stability is given further in the reference frame moving with the constant velocity $V$. We shall consider the case when the planar interface given by equation $z(x)=0$ moves along the $z$-axis of the Cartesian coordinate system $(x, z)$. Then the $2 \mathrm{D}$ steady-state fields of the solute concentration and temperature are obtained from Eqs. (9) and (10) as

$$
\begin{gathered}
\frac{\partial^{2} C}{\partial x^{2}}+\left(1-\frac{V^{2}}{V_{D}^{2}}\right) \frac{\partial^{2} C}{\partial z^{2}}+\frac{V}{D} \frac{\partial C}{\partial z}=0 \\
\frac{\partial^{2} T_{L}}{\partial x^{2}}+\frac{\partial^{2} T_{L}}{\partial z^{2}}+\frac{V}{a_{L}} \frac{\partial T_{L}}{\partial z}=0
\end{gathered}
$$




$$
\frac{\partial^{2} T_{S}}{\partial x^{2}}+\frac{\partial^{2} T_{S}}{\partial z^{2}}+\frac{V}{a_{S}} \frac{\partial T_{S}}{\partial z}=0
$$

Following the standard procedure of the analysis of the morphological stability, let's place harmonic perturbation on the planar interface. The perturbation is described by

$$
z \equiv \phi(x, t)=\delta(t) \sin (\omega x)
$$

where $\delta$ is a small amplitude of perturbation $(|\delta| \ll 1), \omega=2 \pi / \lambda$ is the cyclic frequency with the wavelength, $\lambda$. The response functions on the perturbed interface $\phi(x, t)$, i.e. the temperature, $T_{\phi}$, and solute concentration, $C_{\phi}$, are defined by the following relation

$$
T_{\phi}=T_{m}+m C_{\phi}+\Gamma K
$$

where $m$ is the slope of the liquidus line in the kinetic phase diagram (i.e., phase diagram for nonequilibrium solidification of a binary system), $\Gamma$ is the Gibbs-Thomson coefficient (i.e. the capillary parameter defined by the surface energy of the interface), and $K$ is the mean curvature of the perturbed interface.

For the sake of simplicity of the following analysis, the kinetic term $V / \mu$ (in which $\mu$ is the kinetic coefficient of atomic attachment to the interface) is omitted in Eq. (18). This simplification has no influence on the main results of the present analysis due to the fact that a constant kinetic coefficient does not affect the marginal condition of the front stability $[11,30]$. It has been shown that the kinetic effects influence the interface stability in the case where temperature dependence of the kinetic coefficient [30], or when an optimum stability conjecture for selection of the interface operating state, is used [31].

Considering only the terms of the first order of smallness by the amplitude $\delta$ of perturbation, one can obtain for the curvature of the perturbed interface the following expression

$$
K=\left(\frac{\partial^{2} \phi}{\partial x^{2}}\right)\left(1+\left(\frac{\partial \phi}{\partial x}\right)^{2}\right)^{-3 / 2}=-\delta \omega^{2} \sin (\omega x)
$$

Thus, the system (14)-(19) is the extension of the model for the case of significant deviations from local equilibrium in the solute diffusion field during rapid solidification. In the case of the local equilibrium solute diffusion, i.e. as $V_{D} \rightarrow \infty$, Eq. (14) describes the approximation of Fick's diffusion which has been used in the existing models (see, e.g. Ref. [4]). The system (14)-(19) has been used in the description of local nonequilibrium solidification with planar and non-planar solid-liquid interfaces [19,20,32,33]. With the definition of the functions for solute partitioning $k(V)$ and the liquidus slope $m(V)$, the system 
of Eqs. (14)-(19) can be also applied to the problem of morphological stability of the interfaces in rapid solidification.

\section{Morphological stability}

Within the linear analysis of stability, a solution of equations (14)-(16) on the perturbed interface (17) is described by

$$
\begin{gathered}
C_{\phi}=C_{0}+b \delta(t) \sin (\omega x), \\
T_{\phi}=T_{0}+a \delta(t) \sin (\omega x),
\end{gathered}
$$

where $T_{0}$ and $C_{0}$ are the values for the temperature and solute concentration for the unperturbed planar interface, respectively, and the parameters $b$ and $a$ define the corresponding corrections to the small perturbations on it. Taking into account the small magnitude of perturbations on the planar interface, the perturbed steady-state solution for solute concentration can also be presented in a form proportional to $\delta(t) \sin (\omega x)$. This leads to the following expression

$$
C(x, z)=\bar{C}(z)+F(z) \delta(t) \sin (\omega x)
$$

where $\bar{C}(z)$ is the solute distribution for the planar interface. The function $F(z)$ is defined from Eq. (20). The far-field condition takes the value for the planar interface, i.e. $F(z) \rightarrow 0$ with $z \rightarrow \infty$.

Substitution of Eq. (22) into solute diffusion equation (14) leads to the following approximations:

- in the zero order of magnitude by the amplitude of perturbation $\delta$

$$
\left(1-V^{2} / V_{D}^{2}\right) \frac{d^{2} \bar{C}}{d z^{2}}+\frac{V}{D} \frac{d \bar{C}}{d z}=0
$$

- in the first order of magnitude by the amplitude of perturbation $\delta$

$$
\left(1-V^{2} / V_{D}^{2}\right) \frac{d^{2} F}{d z^{2}}+\frac{V}{D} \frac{d F}{d z}-\omega^{2} F=0 .
$$

A general solution of Eq. (23) has the following form

$$
\bar{C}(z)=C_{1}+C_{2} \exp \left(-\frac{V z}{D\left(1-V^{2} / V_{D}^{2}\right)}\right) .
$$

Solution (25) must be limited at $z \rightarrow \infty$ and it satisfies the following conditions: $\left.\bar{C}\right|_{z=0}=C_{0}$ and $d \bar{C} /\left.d z\right|_{z=0}=G_{C}$, where $G_{C}$ is the concentration gradient at the unperturbed interface. Solution (25) satisfies these conditions in 
the following form

$$
\bar{C}(z)= \begin{cases}C_{0}+\frac{G_{C} D\left(1-V^{2} / V_{D}^{2}\right)}{V}\left[1-\exp \left(-\frac{V z}{D\left(1-V^{2} / V_{D}^{2}\right)}\right)\right], & V<V_{D} \\ C_{0}, & V \geqslant V_{D} .\end{cases}
$$

A general solution of Eq. (24) has the following form

$$
\begin{aligned}
F(z) & =F_{0} \exp \left(-\frac{V+\left(V^{2}+4 D^{2}\left(1-V^{2} / V_{D}^{2}\right) \omega^{2}\right)^{1 / 2}}{2 D\left(1-V^{2} / V_{D}^{2}\right)} z\right) \\
& +F_{1} \exp \left(-\frac{V-\left(V^{2}+4 D^{2}\left(1-V^{2} / V_{D}^{2}\right) \omega^{2}\right)^{1 / 2}}{2 D\left(1-V^{2} / V_{D}^{2}\right)} z\right) .
\end{aligned}
$$

Solution (27) must be limited at $z \rightarrow \infty$ and it takes only the real values for any $\omega$. In this case, Eq. (27) leads to the following particular solution

$$
F(z)= \begin{cases}F_{0} \exp \left(-\frac{\omega_{C} z}{\left(1-V^{2} / V_{D}^{2}\right)^{1 / 2}}\right), & V<V_{D} \\ 0, & V \geqslant V_{D}\end{cases}
$$

Here $F_{0}$ is a constant of integration, and the frequency $\omega_{C}$ is related to the frequency $\omega$ of the perturbation as

$$
\omega_{C}=\frac{V}{2 D\left(1-V^{2} / V_{D}^{2}\right)^{1 / 2}}+\left[\left(\frac{V}{2 D\left(1-V^{2} / V_{D}^{2}\right)^{1 / 2}}\right)^{2}+\omega^{2}\right]^{1 / 2} .
$$

Note that once the interface velocity is equal to or greater than the diffusion speed, $V \geqslant V_{D}$, solution (27) takes the zero values for both constants of integration. $F_{0}=0$ is due to limiting of the solution at the infinite point $z \rightarrow \infty$, and $F_{1}=0$ is for getting the real solution of $F(z)$. Hence, from Eqs. (26) and (28) one can obtain that, with $V \geqslant V_{D}$, the coefficient $b=0$ in Eq. (20).

For obtaining $F_{0}$ it is necessary that Eq. (22), after substitution of Eqs. (26) and (28), must satisfy to Eq. (20) on the perturbed interface (17) in the first order of magnitude. It leads to the following expression

$$
F_{0}=b-G_{C}
$$

Hence, substituting Eqs. (26) and (28)-(30) into Eq. (22), one gets an expres- 
sion for the perturbed field of solute concentration. This yields

$$
C-C_{0}= \begin{cases}\frac{G_{C} D\left(1-V^{2} / V_{D}^{2}\right)}{V}\left[1-\exp \left(-\frac{V z}{D\left(1-V^{2} / V_{D}^{2}\right)}\right)\right] & V<V_{D} \\ +\left(b-G_{C}\right) \delta(t) \sin (\omega x) \exp \left(-\frac{\omega_{C} z}{\left(1-V^{2} / V_{D}^{2}\right)^{1 / 2}}\right), & \\ 0, & V \geqslant V_{D} .\end{cases}
$$

Within the local equilibrium limit $V_{D} \rightarrow \infty$ (i.e. when the interface velocity is much smaller than the diffusion speed, $V \ll V_{D}$ ), solution (31) transforms into solution obtained in Ref. [13] for the case of the local equilibrium solute diffusion transport. Furthermore, as Eq. (31) shows, with the velocities equal to or greater than the solute diffusion speed the concentration does not depend on the interfacial perturbations and is equal to those one at the planar interface. It is known from solution given in Ref. [19] that the solute concentration at the unperturbed planar interface is described by

$$
C(z)-C_{\infty}= \begin{cases}\frac{1-k}{k} C_{\infty} \exp \left(-\frac{V z}{D\left(1-V^{2} / V_{D}^{2}\right)}\right), & V<V_{D} \\ 0, & V \geqslant V_{D}\end{cases}
$$

where $C_{\infty}$ is the solute concentration in the liquid far from the interface (i.e., nominal concentration of the alloy). Then, from Eqs. (31) and (32) it is clear that

$$
C(x, z)=C_{0} \equiv C_{\infty} \text { with } V \geqslant V_{D} .
$$

Therefore, even in the presence of perturbations, a transition to the complete partitionless solidification, $C(x, z)=C_{\infty}$, proceeds in the alloy with the finite velocities $V \geqslant V_{D}$.

A solution of Eqs. (15) and (16) for the heat transfer with the condition (21) is obtained in Ref. [13] and has the following form

$$
\begin{aligned}
T_{L}-T_{0} & =\frac{G_{L} a_{L}}{V}\left[1-\exp \left(-\frac{V z}{a_{L}}\right)\right] \\
& +\left(a-G_{L}\right) \delta(t) \sin (\omega x) \exp \left(-\omega_{L} z\right), \\
T_{S}-T_{0} & =\frac{G_{S} a_{S}}{V}\left[1-\exp \left(-\frac{V z}{a_{S}}\right)\right] \\
& +\left(a-G_{S}\right) \delta(t) \sin (\omega x) \exp \left(\omega_{S} z\right),
\end{aligned}
$$

where $G_{L}$ and $G_{S}$ are the gradients of temperature in the liquid and solid on the unperturbed planar interface, respectively. The frequencies $\omega_{L}$ and $\omega_{C}$ are 
described by

$$
\begin{gathered}
\omega_{L}=\frac{V}{2 a_{L}}+\left[\left(\frac{V}{2 a_{L}}\right)^{2}+\omega^{2}\right]^{1 / 2}, \\
\omega_{S}=-\frac{V}{2 a_{S}}+\left[\left(\frac{V}{2 a_{S}}\right)^{2}+\omega^{2}\right]^{1 / 2} .
\end{gathered}
$$

Using the transport balances (11) and (12), the boundary conditions on the perturbed interface $\phi(x, t)$, Eq. (17), are obtained for the steady-state regime of solidification as follows

$$
\begin{gathered}
-\left.K_{L} \frac{\partial T_{L}}{\partial z}\right|_{\phi}+\left.K_{S} \frac{\partial T_{S}}{\partial z}\right|_{\phi}=Q \tilde{V}, \\
-\left.D\left(1-\tilde{V}^{2} / V_{D}^{2}\right) \frac{\partial C}{\partial z}\right|_{\phi}=\left.(1-k) \tilde{V} C\right|_{\phi} .
\end{gathered}
$$

Here $\tilde{V}$ is the velocity of the perturbed interface. Substitution of the expression $\widetilde{V}=V+(d \delta / d t) \sin (\omega x)$ for the velocity of the perturbed interface into Eqs. (38) and (39) gives the condition of stability regarding the sign of the function $\delta^{-1} d \delta / d t$. The concrete form of the function $\delta^{-1} d \delta / d t$ is obtained as follows.

In the zero order of magnitude by the amplitude $\delta(t)$ of perturbation, substitution of Eqs. (19)-(21) into Eq. (18) gives the following relation

$$
T_{0}=T_{m}+m C_{0},
$$

for the temperature $T_{0}$ and solute concentration $C_{0}$ on the unperturbed planar interface. Eq. (40) is also consistent with the liquidus line in the kinetic diagram of phase state. In the first order of magnitude by the amplitude $\delta(t)$, one gets a relation between coefficients $a$ and $b$ in Eqs. (20) and (21). This yields

$$
a=m b-\Gamma \omega^{2} .
$$

Equation (41) gives a linear relation for the corrections to the temperature and solute concentration on the perturbed interface and uses Gibbs-Thomson effect for the curved interface. For the heat balance at the interface, let us now substitute $\widetilde{V}=V+(d \delta / d t) \sin (\omega x)$ for the interface velocity into the condition (38). Then, in the zero order of magnitude by the amplitude $\delta(t)$, one gets a relation for the temperature gradients and the velocity of the unperturbed planar interface. This is

$$
-K_{L} G_{L}+K_{S} G_{S}=Q V
$$

In the first order of magnitude one gets the following expression

$$
Q \frac{1}{\delta} \frac{d \delta}{d t}=a\left(K_{L} \omega_{L}+K_{S} \omega_{S}\right)+K_{L} G_{L}\left(V / a_{L}-\omega_{L}\right)-K_{S} G_{S}\left(V / a_{S}+\omega_{S}\right),
$$


which gives the change of the amplitude $\delta(t)$ of perturbation in time according to the heat balance. Substituting expression $\widetilde{V}=V+(d \delta / d t) \sin (\omega x)$ into the mass balance (39), one can get in the zero order of magnitude a relation for the gradient of concentration, interface velocity and solute concentration in the following form

$$
-D\left(1-V^{2} / V_{D}^{2}\right) G_{C}=(1-k) V C_{0}
$$

According to Eq. (39), the change of the amplitude $\delta(t)$ of perturbation in time is defined by the first order of smallness by the amplitude of perturbation. This yields

$$
\begin{aligned}
\left(C_{0}-2 D G_{C} V / V_{D}^{2}\right) \frac{1}{\delta} \frac{d \delta}{d t} & =b\left[D \omega_{C}\left(1-V^{2} / V_{D}^{2}\right)^{1 / 2}-(1-k) V\right] \\
& +G_{C}\left[V-D \omega_{C}\left(1-V^{2} / V_{D}^{2}\right)^{1 / 2}\right] .
\end{aligned}
$$

Note that due to introduction of the finite speed $V_{D}$ of solute diffusion into the model, an additional term $-2 D G_{C} V / V_{D}^{2}$ has appeared in the left-hand side of Eq. (45) in comparison to the analysis in [13]. The prefactor $\left(C_{0}-\right.$ $\left.2 D G_{C} V / V_{D}^{2}\right)$ has a positively defined value: one gets $\left(C_{0}-2 D G_{C} V / V_{D}^{2}\right)>0$ due to negative concentrational gradient $G_{C} \leqslant 0$ with $k<1$ at any velocity $V<V_{D}$. Then, to obtain limiting cases, one can take the expression for $G_{C}$ from the balance of solute concentration (44) in the form $G_{C}=$ $-(1-k) V C_{0} /\left(D\left(1-V^{2} / V_{D}^{2}\right)\right)$, and one gets the relation $\left(C_{0}-2 D G_{C} V / V_{D}^{2}\right)=$ $C_{0}\left(1+2(1-k)\left(V^{2} / V_{D}^{2}\right) /\left(1-V^{2} / V_{D}^{2}\right)\right)$. From this it follows the first positive asymptotic: $\left(C_{0}-2 D G_{C} V / V_{D}^{2}\right)=C_{0}>0$ with $V_{D} \rightarrow \infty$. For obtaining the second asymptotic with $V \rightarrow V_{D}$, let us consider the interface velocity in the vicinity of the diffusion speed, i.e. the velocity $V=V_{D}-\varepsilon, 0 \leqslant \varepsilon \ll 1$. Then, for $V \rightarrow V_{D}$, one can obtain the prefactor in the form

$$
\begin{aligned}
& \left.\left(C_{0}-\frac{2 D G_{C} V}{V_{D}^{2}}\right)\right|_{V=V_{D}-\varepsilon}=\left.C_{0}\left(1+\frac{2(1-k(V)) V^{2} / V_{D}^{2}}{1-V^{2} / V_{D}^{2}}\right)\right|_{V=V_{D}-\varepsilon} \\
& =C_{0}\left(1+\left(1-k\left(V_{D}\right)\right) \frac{V_{D}}{\varepsilon}+\left.V_{D} \frac{d k(V)}{d V}\right|_{V=V_{D}}-2\left(1-k\left(V_{D}\right)\right)\right) .
\end{aligned}
$$

From this expression follows two consequences. First, in the case of partition solidification with $V=V_{D}$, one gets $k\left(V_{D}\right) \neq 1$. In this case, the prefactor tends to infinity with $\varepsilon \rightarrow 0$. Second, with the complete solute trapping, $k\left(V_{D}\right)=1$ and $d k(V) / d V \geqslant 0$ one gets for $V \rightarrow V_{D}$ that the prefactor has $a$ positive sign and limited magnitude for the second asymptotic. This yields

$$
0<C_{0}\left(1+\frac{2(1-k(V)) V^{2} / V_{D}^{2}}{1-V^{2} / V_{D}^{2}}\right)=C_{0}\left(1+\left.V_{D} \frac{d k(V)}{d V}\right|_{V=V_{D}}\right)<\infty
$$

Consequently, $\left(C_{0}-2 D G_{C} V / V_{D}^{2}\right)$ is positively defined also for the local equilibrium case $V_{D} \rightarrow \infty$ and local nonequilibrium case $V \rightarrow V_{D}$ if the complete 
solute trapping occurs, $k\left(V_{D}\right)=1$, and influences only the speed of decreasing/increasing of the amplitude of perturbation, but not a selection of the stable mode itself.

From equations (43) and (45), taking into account Eq. (41), the expression for the function $\delta^{-1} d \delta / d t$ can be obtained. The sign of $\delta^{-1} d \delta / d t$ defines the condition of decreasing, $\delta^{-1} d \delta / d t<0$, or increasing, $\delta^{-1} d \delta / d t>0$, of the interfacial perturbation in time. With $\delta^{-1} d \delta / d t=0$ one has the marginal (neutral) stability of the interface $[10,13]$.

\section{Marginal stability}

\subsection{The criterion of marginal stability}

We now consider marginal stability for the neutral stability of a small perturbation on the planar interface, $\delta^{-1} d \delta / d t=0$. From equations (43) and (45), one can obtain expressions for the values of $a$ and $b$. These are

$$
\begin{gathered}
a=K_{L} G_{L} \frac{\omega_{L}-V / a_{L}}{K_{L} \omega_{L}+K_{S} \omega_{S}}+K_{S} G_{S} \frac{\omega_{S}-V / a_{S}}{K_{L} \omega_{L}+K_{S} \omega_{S}}, \\
b= \begin{cases}G_{C} \frac{\omega_{C}-V /\left[D\left(1-V^{2} / V_{D}^{2}\right)^{1 / 2}\right]}{\omega_{C}-(1-k) V /\left[D\left(1-V^{2} / V_{D}^{2}\right)^{1 / 2}\right]}, & V<V_{D}, \\
0, & V \geqslant V_{D} .\end{cases}
\end{gathered}
$$

The system of equations (41), (46), and (47) allows one to obtain a relation for the constant front velocity $V$ and the frequency $\omega$ of a perturbation in the steady-state regime by excluding of $a$ and $b$. This relation can be considered as a final form for the condition of marginal stability.

Let's introduce the following functions of stability

$$
\begin{gathered}
\xi_{L}=\frac{\omega_{L}-V / a_{L}}{K_{L} \omega_{L}+K_{S} \omega_{S}}, \\
\xi_{S}=\frac{\omega_{S}+V / a_{S}}{K_{L} \omega_{L}+K_{S} \omega_{S}}, \\
\xi_{C}= \begin{cases}\frac{\omega_{C}-V /\left[D\left(1-V^{2} / V_{D}^{2}\right)^{1 / 2}\right]}{\omega_{C}-(1-k) V /\left[D\left(1-V^{2} / V_{D}^{2}\right)^{1 / 2}\right]}, & V<V_{D}, \\
0, & V \geqslant V_{D} .\end{cases}
\end{gathered}
$$

The functions $\xi_{L}$ and $\xi_{S}$ in Eqs. (48) and (49) coincide with those derived by Trivedi and Kurz [13]. However, as Eq. (50) shows, the function $\xi_{C}$ of concentrational stability differs from the corresponding function derived previously 
in Ref. [13]. As it follows from Eq. (50), within the local equilibrium limit, $V_{D} \rightarrow \infty$, one gets the special case $\xi_{C}=\left(\omega_{C}-V / D\right) /\left(\omega_{C}-(1-k) V / D\right)$ which coincides with the result given in Ref. [13]. When $V \sim V_{D}$, the function $\xi_{C}$ given by Eq. (50) takes corrections for the relation of the interface velocity, $V$, and diffusion speed, $V_{D}$. With $V \geqslant V_{D}$, the exact equality $\xi_{C}=0$ takes place. This equality is the consequence of solution of the problem of local nonequilibrium solute diffusion which takes into account the finite speed $V_{D}$ in the bulk liquid. Thus, after substituting Eqs. (46) and (47) into Eq. (41) and taking into account Eqs. (48)-(50), one can obtain the criterion of marginal stability. This yields

$$
\begin{cases}\Gamma \omega^{2}+K_{L} G_{L} \xi_{L}+K_{S} G_{S} \xi_{S}-m G_{C} \xi_{C}=0, & V<V_{D} \\ \Gamma \omega^{2}+K_{L} G_{L} \xi_{L}+K_{S} G_{S} \xi_{S}=0, & V \geqslant V_{D}\end{cases}
$$

In the local equilibrium limit, $V_{D} \rightarrow \infty$, criterion (51) transfers into the criterion of marginal stability obtained in Ref. [13] on the basis of a local equilibrium approach to solute diffusion transport. The introduction of the finite diffusion speed, $V_{D}$, into the model leads to the qualitatively new result, which is related to the transition to completely partitionless solidification. As Eq. (51) shows, with the finite interface velocity $V \geqslant V_{D}$, the solute diffusion ahead of the rapid interface is absent [see solution (31)], and the morphological stability is defined by the relation of the stabilizing force $\Gamma \omega^{2}$, due to surface energy, and the contribution $K_{L} G_{L} \xi_{L}+K_{S} G_{S} \xi_{S}$ of temperature gradients, $G_{L}$ and $G_{S}$.

Using criterion (51), one can analyze qualitatively two different situations for solidification when (i) the latent heat is removed from the interface inside the undercooled liquid phase, and (ii) the latent heat is removed from the interface through the solid crystal phase. In case (i), one gets $K_{L} G_{L} \xi_{L}+K_{S} G_{S} \xi_{S}<0$, and the temperature gradient is destabilizing the interface. Therefore, if the absolute morphological stability is not reached by the steady balance $\Gamma \omega^{2}=$ $K_{L} G_{L} \xi_{L}+K_{S} G_{S} \xi_{S}$, the interface is unstable, and the resulting interface may exhibit a cellular-dendritic pattern. In case (ii), with $K_{L} G_{L} \xi_{L}+K_{S} G_{S} \xi_{S}>$ 0 , the total heat flux is directed from the front to the solid phase and the temperature gradient, in addition to the surface energy, stabilizes the form of the solid-liquid interface. In this case, with $V<V_{D}$, the morphological stability is related to a destabilizing action of the force $m G_{C} \xi_{C}$, directly connected with the concentrational gradient $G_{C}$, and the stabilizing force $\Gamma \omega^{2}+K_{L} G_{L} \xi_{L}+$ $K_{S} G_{S} \xi_{S}$, due to the sum of the total positive temperature gradient and the surface energy. With the interface velocity $V \geqslant V_{D}$, solidification leads to the chemically partitionless pattern. A destabilizing action on the front is absent and the interface itself remains linearly stable against any small interfacial perturbation. 


\subsection{Characteristic size for crystal microstructure}

According to the marginal stability hypothesis suggested in Ref. [34] and developed in Refs. [4,14], a characteristic size $R$ selected by crystal microstructure in solidification (e.g., the dendrite tip radius) is related to the critical wavelength, $\lambda$, of interface perturbation as

$$
R \equiv \lambda=\frac{\omega}{2 \pi}
$$

Assuming equality for thermophysical parameters of the liquid and solid, one can obtain characteristic size, $R$, from Eqs. (51) and (52). This yields

- with $V<V_{D}$

$$
R=\left(\frac{\Gamma / \sigma}{m G_{C} \bar{\xi}_{C}-\frac{1}{2}\left(G_{L} \bar{\xi}_{L}+G_{S} \bar{\xi}_{S}\right)}\right)^{1 / 2}
$$

- with $V \geqslant V_{D}$

$$
R=\left(\frac{\Gamma / \sigma}{-\frac{1}{2}\left(G_{L} \bar{\xi}_{L}+G_{S} \bar{\xi}_{S}\right)}\right)^{1 / 2} .
$$

In Eqs. (53) and (54) the following designations are accepted

$$
\begin{gathered}
\sigma=\frac{1}{4 \pi^{2}}, \\
\bar{\xi}_{L}=1-\frac{1}{\left(1+\frac{1}{\sigma P_{T}^{2}}\right)^{1 / 2}}, \\
\bar{\xi}_{S}=1+\frac{1}{\left(1+\frac{1}{\sigma P_{T}^{2}}\right)^{1 / 2}}, \\
\bar{\xi}_{C}=1+\frac{2 k}{1-2 k-\left(1+\frac{1-V^{2} / V_{D}^{2}}{\sigma P_{C}^{2}}\right)^{1 / 2}},
\end{gathered}
$$

with $P_{T}=V R / 2 a$ and $P_{C}=V R / 2 D$ as the thermal and solutal Peclet numbers, respectively. As we noted above, Eq. (54) is true only for solidification in an undercooled liquid, i.e. when the temperature gradient is negative. For the case of solidification in the positive temperature gradient the absolute morphological stability takes place at the interface velocity, $V$, smaller than the diffusion speed, $V_{D}$, in the liquid. 


\section{Absolute stability}

\subsection{Nonisothermal solidification}

Let's consider equation (51) in the limit of large perturbations wavelengths $\lambda \gg 1$, which is true for $\omega \ll 1$. With this condition, from Eqs. (48) and (50) one can yield expansions for the functions $\xi_{L}$ and $\xi_{C}$ in the following form

$$
\xi_{L}=\frac{a_{L}^{2} \omega^{2}}{K_{L} V^{2}}, \quad \xi_{C}= \begin{cases}\frac{\omega^{2} D^{2}\left(1-V^{2} / V_{D}^{2}\right)}{k V^{2}}, & V<V_{D} \\ 0, & V \geqslant V_{D}\end{cases}
$$

Substituting these expressions into the criterion of marginal stability (51) at $G_{S}=0$, we get the criterion of absolute morphological stability for the planar front. This criterion can be written in the form of the following nonlinear equation for the velocity, $V_{A}$, of absolute stability:

$$
V_{A}=V_{A}^{T}(V)+V_{A}^{C}(V)
$$

where

$$
V_{A}^{T}(V)=-\frac{a_{L}}{\Gamma}\left(\frac{a_{L} G_{L}}{V}\right)
$$

is the velocity of absolute thermal stability, and

$$
V_{A}^{C}(V)=\frac{D}{\Gamma k}\left(\frac{D\left(1-V^{2} / V_{D}^{2}\right) m G_{C}}{V}\right)<V_{D}
$$

is the velocity of absolute chemical stability.

The velocity, $V_{A}^{T}$, of absolute thermal stability, Eq. (61), shows the relationship between the contribution of the temperature gradient, $G_{T}$, and capillary parameter, $\Gamma$. With the negative temperature gradient, $G_{L}<0$, the range of morphological stability shrinks. Conversely, the positive temperature gradient, $G_{L}>0$, extends the range of the velocities at which the planar front is linearly stable. The velocity, $V_{A}^{C}$, of absolute chemical stability, Eq. (62), defines the contribution of the concentrational gradient, $G_{C}$, and capillary parameter $\Gamma$. This velocity is always less than the diffusion speed, $V_{A}^{C}<V_{D}$, because the velocity $V_{A}^{C}$ is defined by the steady balance between surface tension, given by the capillary parameter $\Gamma$, and the gradient $G_{C}$ of solute concentration, existing up to the completion of solute diffusion. Consequently, Eqs. (60)-(62) exhibit a competition of destabilizing and stabilizing forces. With the velocity, $V<V_{A}$, the planar interface is perturbed with a possible originating of the cellular-dendritic patterns. As the solidification velocity increases, $V>V_{A}$, the planar interface becomes morphologically stable against any small perturbation of its form. 
To clarify contributions from both thermal and solute diffusion on the absolute stability of the interface, we define the gradients in Eqs. (61)-(62) in explicit form. For the thermal and concentrational gradients at the unperturbed planar interface we use a solution of the local-nonequilibrium problem [19]. From the solution, one gets

$$
G_{L}=-\frac{T_{Q} V}{a_{L}}, \quad G_{C}= \begin{cases}-\frac{(1-k) V C_{\infty}}{k D\left(1-V^{2} / V_{D}^{2}\right)}, & V<V_{D} \\ 0, & V \geqslant V_{D}\end{cases}
$$

where $T_{Q}$ is a unit of undercooling equal to $Q / \chi_{L}$, and $C_{\infty}$ is the solute concentration in the liquid far from the interface. From the second expression in Eq. (63), it follows that when the solute diffusion is absent ahead of the interface with $V \geq V_{D}$, the gradient of the solute concentration is zero exactly. Substitution of Eq. (63) into Eqs. (60)-(62) gives the expression for the absolute stability of the interface. This yields

$$
V_{A}=\left\{\begin{array}{ll}
V_{A}^{T}+V_{A}^{C}, & V<V_{D}, \\
V_{A}^{T}, & V \geqslant V_{D},
\end{array}= \begin{cases}\frac{a_{L}}{\Gamma} \Delta T_{T}+\frac{D}{\Gamma k} \Delta T_{C}, & V<V_{D}, \\
\frac{a_{L}}{\Gamma} \Delta T_{T}, & V \geqslant V_{D},\end{cases}\right.
$$

where $\Delta T_{T}=T_{Q}$ is the thermal undercooling, which is necessary for solidification with the planar interface on the thermal scale, and $\Delta T_{C}=(k-1) m C_{\infty} / k$ is the constitutional undercooling, which is necessary for solidification with the planar interface on the scale of solute diffusion. Additionally, $\Delta T_{C}$ is the nonequilibrium temperature interval of solidification between liquidus and solidus lines in the kinetic diagram of phase state.

The criterion $V_{A}^{T}=a_{L} \Delta T_{T} / \Gamma$ in Eq. (64) is the same as that which has been obtained by Trivedi and Kurz [13] using the advanced model for large growth velocities. The criterion $V_{A}^{C}=D \Delta T_{C} / \Gamma=D(k-1) m C_{\infty} /\left(\Gamma k^{2}\right)$ in Eq. (64) is similar to that which has been obtained by Mullins and Sekerka [10] for the case of small growth velocities, and re-derived by Trivedi and Kurz [13] for the case of rapid solidification. In addition to this treatment, by introducing the finite speed, $V_{D}$, into the model we reach a qualitative new result. At the finite velocity, $V \geq V_{D}$, due to the absence of the solute diffusion $\left[G_{C}=0\right.$, Eq. (63)], the interval between nonequilibrium liquidus and solidus lines is equal to zero, $\Delta T_{C}=0$. These lines converge in the kinetic phase diagram with $V \geq V_{D}$ [19], and the absolute stability of the planar interface is defined only by the undercooling $\Delta T_{T}$, and relation between the thermal diffusivity, $a_{L}$, and capillary parameter, $\Gamma$, Eq. (64). 


\subsection{Isothermal solidification}

In the analysis of the criterion of marginal stability (51), a special interest is given to the case in which the form of the interface is defined by the competition between stabilizing force, $\Gamma \omega^{2}$, due to surface energy, and destabilizing force, $m G_{C} \xi_{C}$, due to gradient, $G_{C}$, of solute concentration. Assuming the zero temperature gradient, $G_{L}=0$, in Eq. (60), one can obtain an explicit expression for the condition of absolute chemical stability of the interface, $V_{A}=V_{A}^{C}$. Using the expression for $G_{C}$ from (63), one gets

$$
V_{A}=\frac{m D(k-1) C_{\infty}}{\Gamma k^{2}}<V_{D}
$$

The form of this expression coincides with the expression given for the case of local equilibrium solute diffusion transport at $V_{D} \rightarrow \infty$ and $V \ll V_{D}$ [13]. However, a final form of the function $V_{A}\left(C_{\infty}\right)$ is defined by the functions of solute partitioning, $k(V)$, and the slope, $m(V)$, of liquidus line in the kinetic phase diagram. The behavior of theses functions is rather different for the cases of local equilibrium and local nonequilibrium solute diffusion $[19,35]$.

\section{Discussion}

In the first part of the discussion, we synthesize our system of equations to give the self-consistent model, which is adopting the deviation from local equilibrium in the solute diffusion field for all functions. We discuss the solute partitioning functions and the expression for the slope of kinetic liquidus which take into account the deviation from local equilibrium, both at the interface and in the bulk liquid around the interface. Then, in the second part of the discussion, we present a quantitative evaluation of the discrepancies between the present model and the model in which the local nonequilibrium is taken only at the interface. These are compared with experimental data on the absolute stability of the planar interface.

\subsection{Solute partitioning and kinetic liquidus}

The boundary condition for solute diffusive transport can be given on the basis of the continuous growth model (CGM) [36]. The CGM gives the solute partitioning function at the solid-liquid interface, which, in the dilute solution 
approximation, is described by $[36,37]$

$$
k(V)=\frac{k_{e}+V / V_{D I}}{1+V / V_{D I}}
$$

where $V_{D I}$ is the speed of diffusion at the interface, and $k_{e}$ is the value of the equilibrium partition coefficient with $V \rightarrow 0$. One of the deficiencies of the function (66) is the difficulty to describe of the complete solute trapping at the finite interface velocity, i.e. it predicts $k \rightarrow 1$ only with $V \rightarrow \infty$. However, as it has been shown in numerous experiments (see, e.g., Refs. [38,39]), a transition to partitionless solidification occurs at a finite solidification velocity. Furthermore, the molecular dynamic simulation has shown [40] that the transition to the complete solute trapping is observed at finite crystal growth velocity. Therefore, in addition to Eq. (66), a generalized function for solute partitioning, in the case of local nonequilibrium solute diffusion within the approximation of a dilute alloy, has been introduced [41]. This yields

$$
k(V)= \begin{cases}\frac{k_{e}\left(1-V^{2} / V_{D}^{2}\right)+V / V_{D I}}{1-V^{2} / V_{D}^{2}+V / V_{D I}}, & V<V_{D}, \\ 1, & V \geqslant V_{D},\end{cases}
$$

where $V_{D I}$ is the interfacial diffusion speed with $V_{D I} \leq V_{D}[20,41]$. In the local equilibrium limit, i.e. when the bulk diffusive speed is infinite, $V_{D} \rightarrow \infty$, expression (67) reduces to the function $k(V)$, that takes into account the deviation from local equilibrium at the interface only, Eq. (66). In addition to the previous model [36,37], the function $k(V)$ described by Eq. (67) includes the deviation from local equilibrium not only at the interface (introducing interfacial diffusion speed $V_{D I}$ ), but also in the bulk liquid (introducing diffusive speed $V_{D}$ in bulk). As Eq. (67) shows, the complete solute trapping, $k(V)=1$, proceeds at $V=V_{D}$.

A thermodynamic approach applied to the solidification of a binary system [42] provided two models for the solute trapping with and without solute drag $[23,43]$. These models give a shift from local equilibrium at the interface which can be expressed in unified form for the slope, $m(V)$, of kinetic liquidus by the following equation

$$
m(V)=\frac{m_{e}}{1-k_{e}}\left\{1-k+\left[k+(1-k) \delta_{0}\right] \ln \left(\frac{k}{k_{e}}\right)\right\} .
$$

Here $\delta_{0}=0$ is for the model of solute trapping without solute drag and $\delta_{0}=1$ is for the model of solute trapping with solute drag. Introducing Eq. (66) into Eq. (68), one obtains the constant liquidus slope, $m$, (independent of $V$ ) only with the infinite interface velocity, $V \rightarrow \infty$.

Using the results of the local nonequilibrium thermodynamic analysis [35], one arrives to the slope of the liquidus line in the following form 


$$
m(V)= \begin{cases}\frac{m_{e}}{1-k_{e}}\left\{1-k+\ln \left(\frac{k}{k_{e}}\right)+(1-k)^{2} \frac{V}{V_{D}}\right\}, & V<V_{D}, \\ \frac{m_{e} \ln k_{e}}{k_{e}-1}, & V \geqslant V_{D} .\end{cases}
$$

With $V<V_{D}$, the function $m(V)$ includes the function described by Eq. (68) for the solute trapping with solute $\operatorname{drag}\left(\delta_{0}=1\right)$ and the additional term $(1-k)^{2} V / V_{D}$. This term arises from the analysis of the Gibbs free energy, taking into account local nonequilibrium solute diffusion around the interface. It is necessary to note that the function $m(V)$ described by Eq. (69) plays a crucial role for self-consistency of the theory of local nonequilibrium solidification. This form of the function has been used in a self-consistent model for rapid dendritic growth and gave quantitative agreement with experimental data on kinetics of alloy solidification $[20,32]$. In particular, the self-consistent dendritic growth model, including Eq. (69), predicts the breakpoint at $V=V_{D}$ with good agreement of data on a number of investigated alloys. Furthermore, Eq. (69) gives us the ability to describe a transition from the growth kinetics, with solute drag effect at small and moderate solidification velocities (arising with the developed solute profile ahead of the interface), to the growth kinetics without solute drag at high solidification velocities (with the degeneration of the solute profile ahead the interface) $[35,44]$. Thus, Eqs. (67) and (69) close the system of equations (14)-(19) for the self-consistent analysis of morphological stability.

\subsection{Comparison with experimental data}

To discuss the results obtained for the interfacial stability, we now compare the model predictions for the absolute stability condition (65) in both cases of solidification, namely, with local equilibrium solute diffusion and with local nonequilibrium solute diffusion transports. Substituting functions (66)-(69) into Eq. (65), we analyze the absolute stability of the planar interface for different velocities. We stress two important points regarding the choice of the expression for the slope of kinetic liquidus given by Eq. (68). First, the result on rapid dendritic growth [20,32] gives evidence to the confluence of all model predictions at small undercoolings and low growth velocities. Disagreement of the kinetic curves begins from the undercooling approximately corresponding to the undercooling for the absolute chemical stability at moderate growth velocities. Therefore, our present discussion for interfacial stability is limited by the moderate interface velocities, when the model's predictions (with or without taking local nonequilibrium in bulk liquid) begin to disagree. Second, in this region of velocities, the local nonequilibrium approach to rapid solidification gives a similar result with the model, which takes into account 
the deviation from local equilibrium only at the interface, with the solutedrag effect (see the analysis presented in Refs. $[35,44]$ ). This fact is due to the existence of the developed solute profile ahead of the interface at small and moderate velocities, when the solute drag may appear at the interface. Consequently, in order to evaluate the disagreement between the model and experimental data, we choose the expression for the slope of the kinetic liquidus, which adopts the solute-drag effect, i.e. it is chosen in the following calculations where $\delta_{0}=1$ for Eq. (68).

Using parameters of an Al-Fe alloy from Table 1, one can calculate the curve for critical concentration, $C_{\infty}(V)$, which gives a threshold for interface instability. As it can be seen from Fig. 3, two regions of the interfacial existence may occur: the planar interface is absolutely stable below the curves and the interface breaks down in the regions above the curves given by the functions $C_{\infty}(V)$. In comparison with the model with the local equilibrium diffusion and deviation from local equilibrium at the interface only [Eqs. (65, (66), and (68)], the present model for interface stability with the deviation from local equilibrium, both at the interface and in the bulk liquid [Eqs. (65), (67, and (69)], defines a curve $C_{\infty}(V)$ which is limited by the diffusion speed $V_{D}$ for morphological stability of the interface. This limit exists due to a steady balance between the stabilizing capillary force and the destabilizing force defined by the concentrational gradient, which still acts on the interface up to the finishing of diffusion, i.e. until the point $V=V_{D}$.

For a quantitative comparison of the model predictions we have chosen experimental results on interface stability during rapid solidification of a $\mathrm{Si}-\mathrm{Sn}$ alloy as presented by Hoglund and Aziz in Ref. [46]. These authors measured a critical concentration of Sn for interface breakdown in a steady-state solidification after pulsed laser melting. Using the parameters of the Si-Sn alloy from Table 1 , the model predictions for the function $C_{\infty}(V)$ are compared quantitatively with experimental results from Ref. [46]. As is shown in Fig. 4, the present model for interface stability with the local nonequilibrium diffusion [Eqs. (65), (67], and (69)] gives a satisfactory comparison with the experiment. At the concentration $\mathrm{Sn}=0.02$ atomic fraction (see the extreme right experimental point in Fig. 4), the discrepancy between the model with local equilibrium solute diffusion (curve 1 in Fig. 4) and experiment gives the value of $38.90 \%$ (see Table 2). At the same alloy's concentration, the present model (curve 2 in Fig. 4) gives the discrepancy with experiment of $16.93 \%$ (see Table 2). Consequently, even better comparison with the available experimental data can be obtained with using the present model of local nonequilibrium solidification. 


\section{Conclusions}

Morphological stability of the planar interface in rapid solidification of nonisothermal binary system has been considered. We have taken into account the fact that the high rate of solidification process leads to the absence of a local thermodynamic equilibrium in the solute diffusion field and at the solid-liquid interface. The presently developed model is self-consistent: the main governing equations, Sec. 2, and the interface conditions for solute trapping and kinetic liquidus, Sec. 6, are consistent with the formalism of extended thermodynamic approach to rapid solidification [35]. Using the model of local nonequilibrium rapid solidification, our analysis of morphological stability extends the previous analysis of Trivedi and Kurz [13], which has been performed to advance the treatment of Mullins and Sekerka [10] to the case of rapid solidification. The main outcomes of this analysis are summarized as follows.

(i) For the velocities equal to or greater than the diffusion speed, $V \geq V_{D}$, from solutions (31)-(33) it follows that the field of concentration does not depend on a form of the interfacial perturbation and it is equal to the initial (nominal) concentration, $C(x, z)=C_{\infty}$. This result is in agreement with the previous results for planar and parabolic interfaces [19,33]. Solutions (31)(33) has a clear physical meaning: a source of concentrational disturbances, i.e. the perturbed interface, cannot disturb a binary liquid ahead of itself if the interface velocity is equal to or greater than the maximum speed of these disturbances.

(ii) The obtained criterion of the marginal stability, Eq. (51), defines a wavelength of perturbation for the neutral stability. For $V<V_{D}$, the neutral stability is defined by a balance of the stabilizing force, due to surface energy, destabilizing force, due to concentrational gradient $G_{C}$, and the contribution of temperature gradients $G_{L}$ and $G_{S}$. Qualitatively, a new result can be obtained from the criterion for the front velocity of $V \geq V_{D}$, i.e. for an absence of the solute diffusion ahead of the interface. As it follows from the second equation in Eq. (51), the morphological stability of the interface is defined only by the relation between the thermodynamic stabilizing force, due to the surface tension, and the driving force of the morphological instability, due to the negative thermal gradient in the undercooled liquid. In the case of directional solidification with the positive thermal gradient, the destabilizing action on the interface is absent at $V \geq V_{D}$, and the interface remains linearly stable against small perturbations of its form.

(iii) Absolute stability of the planar interface is considered as a steady balance between destabilizing force (due to the concentrational gradient), the thermal contribution (due to the thermal gradient), and the stabilizing force (due to surface tension). The velocity, $V_{A}$, of the absolute interface stability is obtained 
as a sum of the velocity, $V_{A}^{T}$, for thermal stability and velocity, $V_{A}^{C}$, for chemical stability defined by Eq. (64). $V_{A}^{C}$ is the same as what was obtained by Mullins and Sekerka [10] for the case of small growth velocities. It was re-derived by Trivedi and Kurz [13] for the case of rapid solidification. Introduction of the finite speed, $V_{D}$, into the model gives the qualitatively new result: with the absence of the solute diffusion at $V \geq V_{D}$, the absolute stability of a planar interface is defined only by the thermal undercooling and relation between the thermal diffusivity, $a_{L}$, and capillary parameter, $\Gamma$, Eq. (64). For an isothermal solidification, the present analysis shows the limiting boundary equals to the diffusion speed, $V_{D}$, for the region of morphological instability [see Eq. (65) and Fig. 3].

(iv) The predictions of the present model for the critical concentration above which a planar interface becomes unstable [see Eqs. (65), (67), and (69)] are compared with the previous model, which adopts the deviation from local equilibrium at the interface only [see Eqs. (65), (66), and (68)], and with the experimental data obtained for solidification of the $\mathrm{Si}-\mathrm{Sn}$ alloy [46]. As it is shown in Fig. 4, the present model is able to describe experimental data satisfactorily in a whole region of the interface velocities investigated. From numerical evaluation of the theoretical predictions summarized in Table 2, it follows that better agreement with experiment is obtained with using the present model of local nonequilibrium solidification.

\section{Acknowledgements}

This work was performed with support from the DFG Schwerpunktprogramm 1120 under Research Projects He 1601/13 and Ne 822/2-1. 


\section{References}

[1] Flemings MC. Solidification Processing. New York: McGraw-Hill, 1974.

[2] Chernov AA. Modern Crystallography. Vol.III. In: Cardona M, Fulde P, Queisser H-J, editors. Springer Series in Solid-State Science. Vol.36. Berlin: Springer, 1984.

[3] Vicsek T. Fractal Growth Phenomena, 2nd Edition. Singapore: World Scientific, 1992.

[4] Kurz W, Fisher DJ. Fundamentals of Solidification, 3rd Edition. Aedermannsdorf: Trans Tech Publications, 1992.

[5] Galenko PK, Zhuravlev VA. Physics of Dendrites. Singapore: World Scientific, 1994.

[6] Tiller WA, Jackson KA, Rutter JW, Chalmers B. Acta Metall 1953;1:428.

[7] Sahm PR, Jones H, Adam CM, editors. Science and Technology of the Undercooled Melt. Dordrecht: Martinus Nijhoff, 1986.

[8] Galenko PK, Krivilyov MD. In: Novak MM and Dewey TG, editors. Fractal Frontiers. Singapore: World Scientific, 1997. p.411.

[9] Mullins WW, Sekerka RF. J Appl Phys 1963;34:323.

[10] Mullins WW, Sekerka RF. J Appl Phys 1964;35:444.

[11] Coriell SR, McFadden GB. In: Hurl DTJ, editor. Handbook of Crystal Growth, Vol.1a. Amsterdam: Elsevier, 1993. p.785.

[12] Davis SH. Theory of Solidification. Cambridge, UK: Cambridge University Press, 2001.

[13] Trivedi R, Kurz W. Acta Metall 1986;34:1663.

[14] Kurz W, Giovanola B, Trivedi R. Acta Metall 1986;34:823.

[15] Galenko PK. Kristallografiya 1993;38(6):238 [Crystallogr Rep 1993;38:836].

[16] Herlach DM. Mater Sci Eng R 1994;12:177.

[17] Glansdorff P, Prigogine I. Thermodynamic Theory of Structure, Stability and Fluctuations. New York: Wiely, 1971.

[18] Prigogine I. Physica 1949;15:272.

[19] Galenko P, Sobolev S. Phys Rev E 1997;55:343.

[20] Galenko PK, Danilov DA. Phys Lett A 1997;235:271.

[21] Lee SC, Hwang CC, Hsieh JY, Lee KY. J Cryst Growth 1999;200:305.

[22] Cantor B. In: Ref. [7]. p.3. 
[23] Boettinger WJ, Coriell SR. In: Ref. [7]. p.81.

[24] Galenko PK, Krivilyov MD, Lad'yanov VI, Osetrov MV. Application of the Model of Locally Nonequilibrium Solidification to the Process of Structure Formation in Alloys Rapidly Quenched by Spinning. Preprint No.827-VOO. Moscow: VINITI, 2000.

[25] Galenko PK, Danilov DA. Phys Lett A 2000;278:129.

[26] Jou D, Camacho J, Grmela M. Macromolecules 1991;24:3597.

[27] Jou D, Casas-Vazquez J, Lebon G. Rep Prog Phys 1988;51:1005.

[28] Jou D, Casas-Vazquez J, Lebon G. Rep Prog Phys 1999;62:1035.

[29] Jou D, Casas-Vazquez J, Lebon G. Extended Irreversible Thermodynamics, 2nd Edition. Berlin: Springer, 1996.

[30] Li J, Yang G, Zhou Y. Mater Res Bulletin 2000;35:1775.

[31] Sekerka RF. J Cryst Growth 1995;154:377.

[32] Galenko PK, Danilov DA. J Cryst Growth 1999;197:992.

[33] Galenko PK, Danilov DA. Phys Lett A 2000;272:207.

[34] Langer JS, Müller-Krumbhaar H. Acta Metall 1978;26:1681.

[35] Galenko P. Phys Rev B 2002;65:144103.

[36] Aziz MJ, Kaplan T. Acta Metall 1988;36:2335.

[37] Aziz MJ. J Appl Phys 1982;53:1158.

[38] Miroshnichenko IS. Quenching From the Liquid State. Moscow: Metallurgia, 1982.

[39] Eckler K, Cochrane RF, Herlach DM, Feuerbacher B, Jurisch M. Phys Rev B 1992;45:5019.

[40] Cook SJ, Clancy P. J Chem Phys 1993;99:2175.

[41] Sobolev SL. Phys Stat Sol A 1996;156:293.

[42] Baker JC, Cahn JW. In: Hughel TJ, Bolling GF, editors. Solidification. Metals Park, OH: American Society of Metals, 1971. p.23.

[43] Aziz MJ, Boettinger WJ. Acta Metall Mater 1994;42:527.

[44] Galenko PK. Mater Sci Eng 2003 [in press].

[45] Gremaud M, Carrard M, Kurz W. Acta Metall Mater 1990;38:2587.

[46] Hoglund DE, Aziz MJ. In: Thompson MO, Aziz MJ, Stephenson GB, editors. Kinetics of Phase Transformations. Materials Research Society Symposia Proceedings, Vol.205. (Pittsburgh, PA: MRS, 1992). p.325. 
Table 1

Physical parameters used in calculations of the limit of absolute stability of the planar interface in solidifying binary alloys

\begin{tabular}{lllll}
\hline \hline Parameter & & & $\mathrm{Al}-\mathrm{Fe}$ & $\mathrm{Si}-\mathrm{Sn}$ \\
\hline Diffusion coefficient & $D_{L}$ & $\mathrm{~m}^{2} / \mathrm{s}$ & $1.7 \times 10^{-9}(*)$ & $2.5 \times 10^{-8}(* *)$ \\
Partition coefficient & $k_{e}$ & - & $0.03(*)$ & $0.016(* *)$ \\
Liquidus slope & $m_{e}$ & $\mathrm{~K} / \mathrm{at} . \%$ & $-7.3(*)$ & $-4.6(* *)$ \\
Gibbs-Thomson coefficient & $\Gamma$ & $\mathrm{K} \cdot \mathrm{m}$ & $1 \times 10^{-7}(*)$ & $1.3 \times 10^{-7}(* *)$ \\
Interface diffusion speed & $V_{D I}$ & $\mathrm{~m} / \mathrm{s}$ & 7 & $17(* *)$ \\
Diffusion speed in bulk liquid & $V_{D}$ & $\mathrm{~m} / \mathrm{s}$ & 10 & 17.5 \\
\hline
\end{tabular}

$(*)$ - Data taken from Ref. [45].

$(* *)$ - Data taken from Ref. [46].

Table 2

Discrepancy between theoretical predictions and experiment for the absolute chemical stability of the planar interface in the Si-0.02(at.fraction)Sn alloy

\begin{tabular}{llcc}
\hline \hline Absolute chemical stability & $\begin{array}{c}\text { Velocity } \\
(\mathrm{m} / \mathrm{s})\end{array}$ & $\begin{array}{c}\text { Definition } \\
\text { of discrepancy }\end{array}$ & $\begin{array}{c}\text { Value } \\
\text { of discrepancy }(\%)\end{array}$ \\
\hline Local equilibrium solute diffusion, & & \\
Eqs. (65), (66), and (68) & $V_{A}^{(1)}=15.5$ & $\frac{V_{A}^{(1)}-V_{A}^{(e x p)}}{V_{A}^{(1)}} 100 \%$ & 38.90 \\
\hline Local nonequilibrium solute diffusion, & & \\
Eqs. (65), (67), and (69) & $V_{A}^{(2)}=11.4$ & $\frac{V_{A}^{(2)}-V_{A}^{(e x p)}}{V_{A}^{(2)}} 100 \%$ & 16.93 \\
\hline Experiment, Ref. [46] & $V_{A}^{(e x p)}=9.47$ & - \\
\hline \hline
\end{tabular}


Figure captions to the article "Linear morphological stability analysis of the solid-liquid interface in rapid solidification of a binary system".

Fig. 1. Morphological diagram for solidification of binary systems which is illustrating the microstructural transitions "planar front" $\rightarrow$ "cellular structure" $\rightarrow$ "dendrites" $\rightarrow$ "cellular structure" $\rightarrow$ "planar front", with the increasing of the solidification velocity, $V$. Here $V_{C}$ is the velocity given by the criterion of constitutional undercooling, and $V_{A}$ is the velocity for absolute morphological stability of the interface.

Fig. 2. Optical micrograph of longitudinal through-thickness section of a melt-spun ribbon of Ni-18 at.\% B [24]. The crystal microstructure exhibits a transition from planar interface with solute segregation-free to cellular-dendritic patterns. The transition proceeds due to decreasing of the interface velocity from $V>V_{A}$ up to $V<V_{A}$. Wheel surface at bottom of micrograph.

Fig. 3. Velocity $V_{A}$ of absolute chemical stability versus solute concentration $C_{\infty}$ for Al-Fe alloy. Dashed curve corresponds to solution of Eqs. (65), (66), and (68) with solute-drag effect. Solid curve corresponds to solution of Eqs. (65), (67), and (69). Dashed-dotted line, $V=V_{D}$, represents the limiting velocity for the absolute interface stability.

Fig. 4. Critical concentration, $C_{\infty}$, above which planar interface is unstable. Experimental points correspond to solidification of the $\mathrm{Si}-\mathrm{Sn}$ alloy [46]. Circles are taken from measurements performed on bulk single crystal $\mathrm{Si}(100)$, and squares are taken from measurements using Sn-implanted Si-on-sapphire (SOS) samples. Curves are given by the models for interfacial absolute stability: dashed - with local equilibrium diffusion and solute drag effect, Eqs. (65), (66), and (68); solid - with the local nonequilibrium diffusion, Eqs. (65), (67), and (69). Dashed-dotted line, $V=V_{D}$, represents the limiting velocity for the absolute interface stability. 


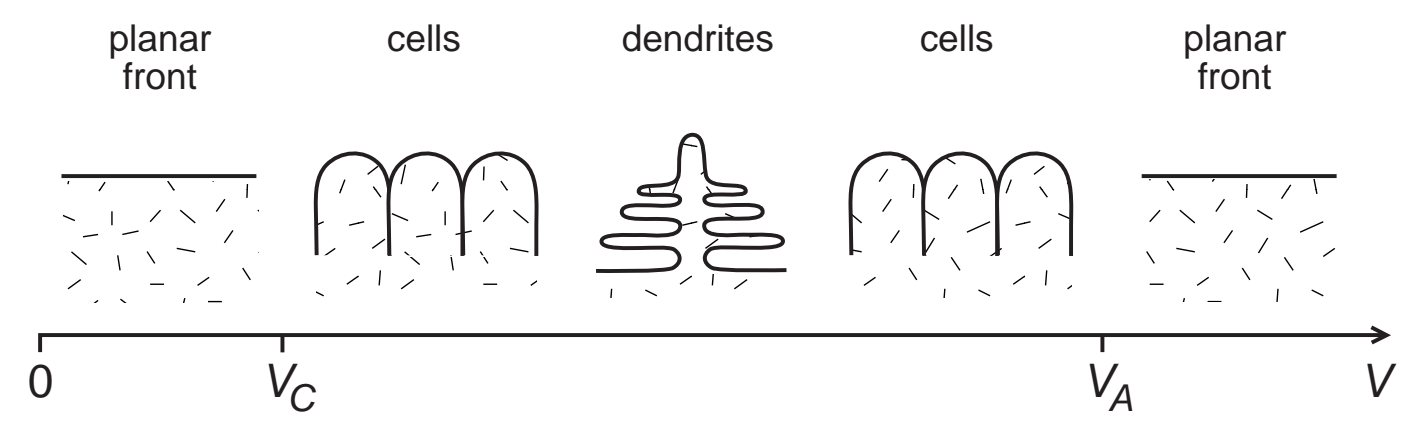

Figure 1 


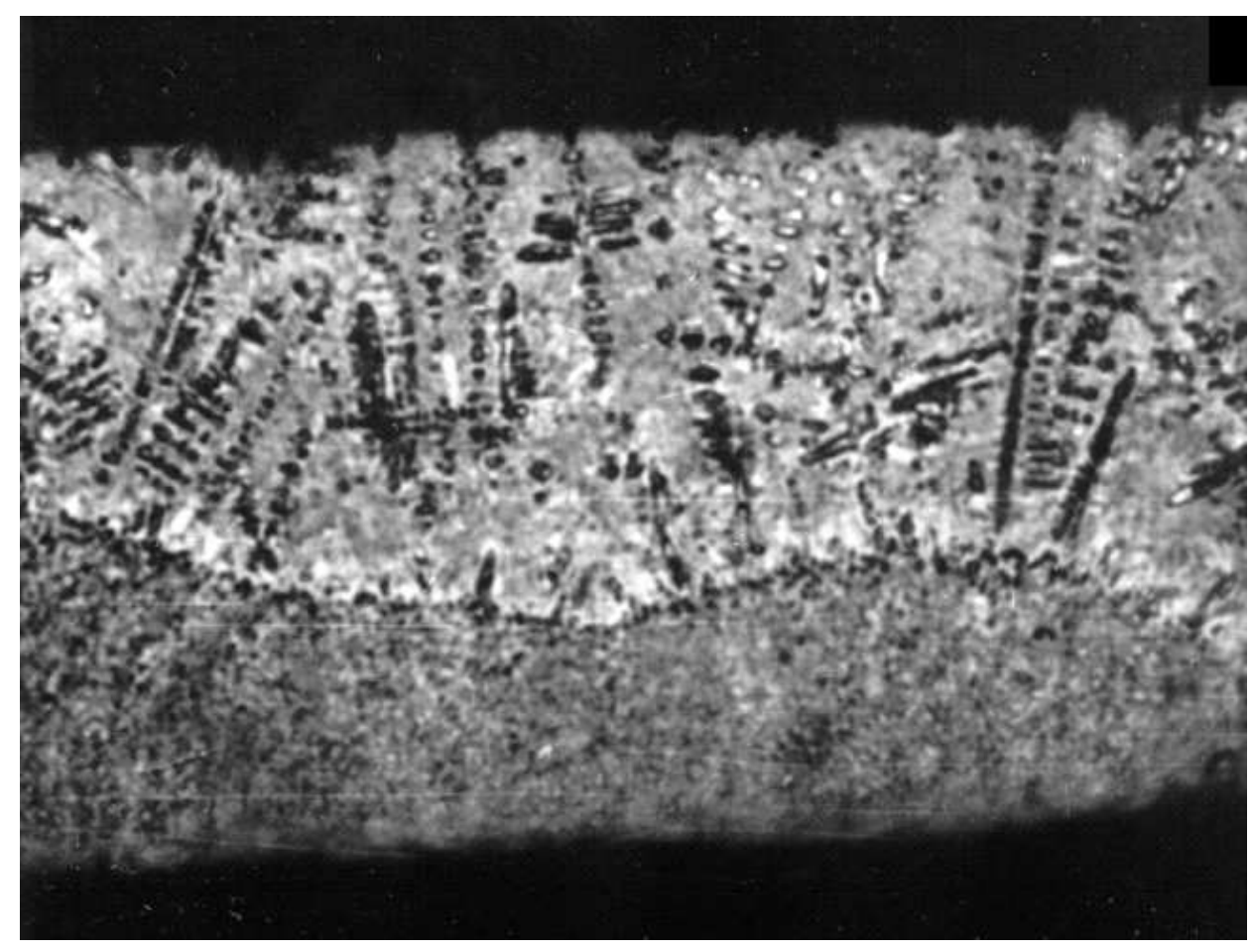

Figure 2 


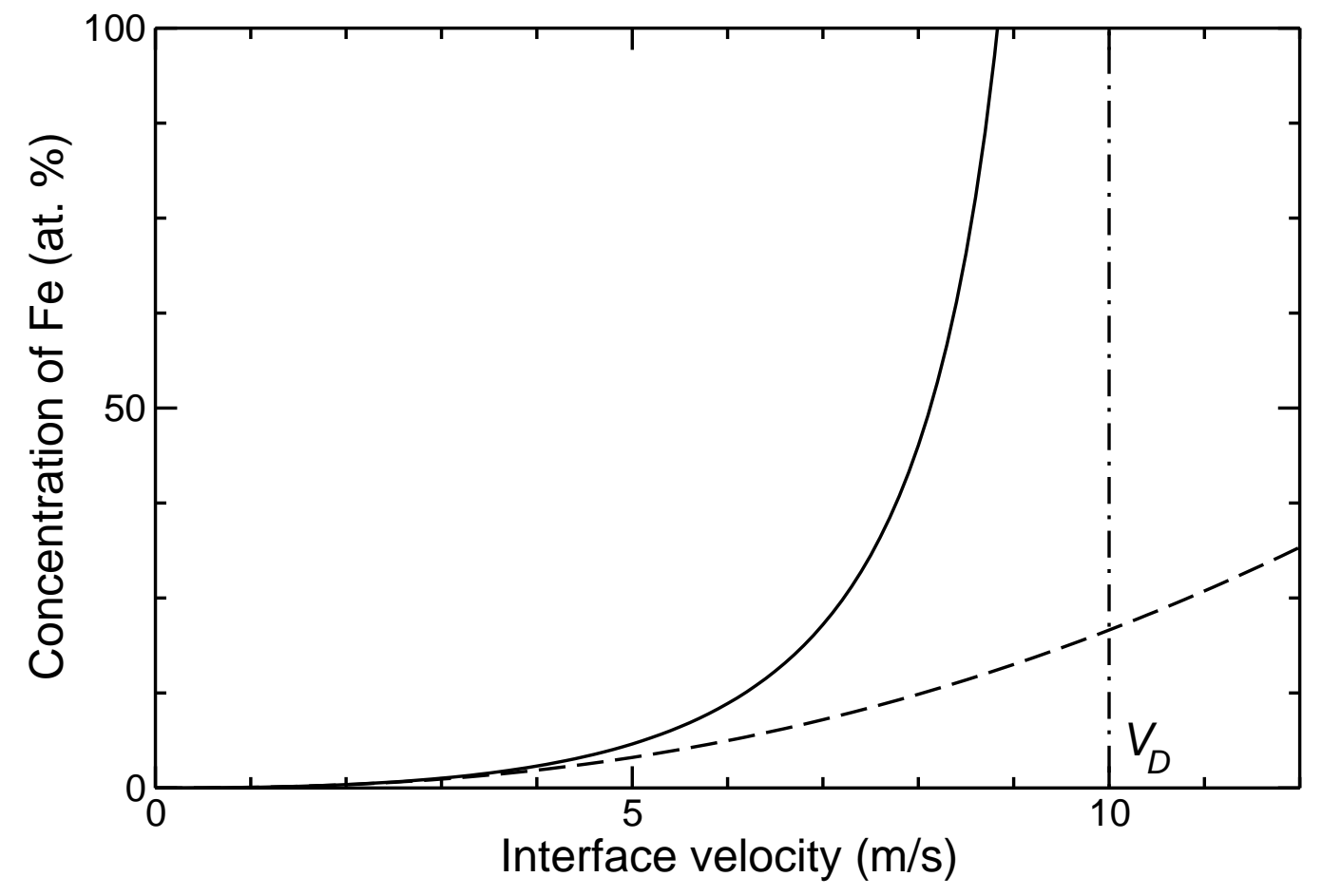

Figure 3 


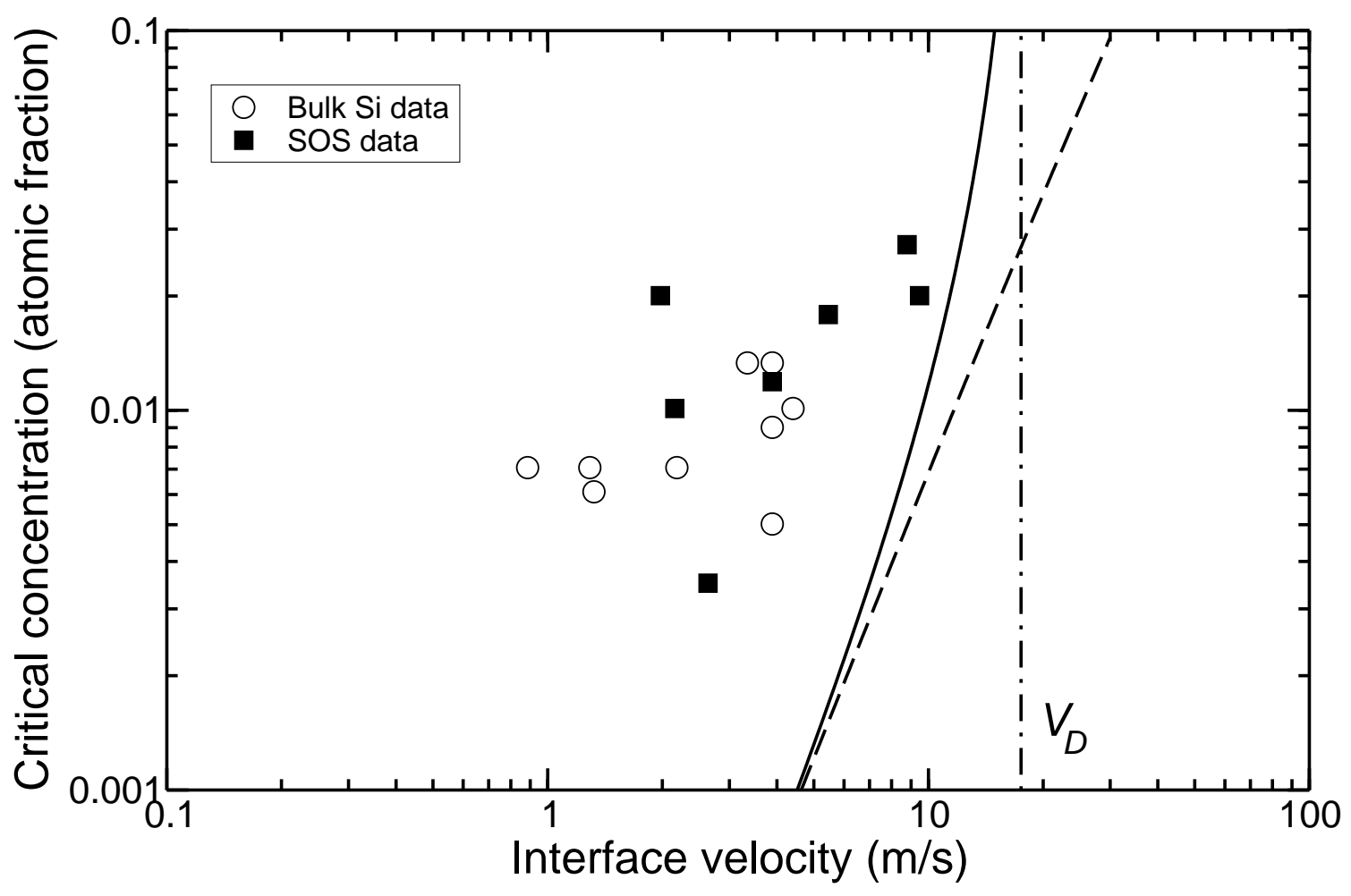

Figure 4 